\title{
Impacto da saúde na qualidade de vida e trabalho de docentes universitários de diferentes áreas de conhecimento
}

\author{
Impact of health on quality of life and quality of working life of \\ university teachers from different areas of knowledge
}

Hugo Machado Sanchez (https://orcid.org/0000-0001-5389-9783) ${ }^{1}$

Eliane Gouveia de Morais Sanchez (http://orcid.org/0000-0003-1291-7652) ${ }^{1}$

Maria Alves Barbosa (http://orcid.org/0000-0002-0861-9655) ${ }^{2}$

Ednaldo Carvalho Guimarães (http://orcid.org/0000-0001-8328-9687) ${ }^{3}$

Celmo Celeno Porto (http://orcid.org/0000-0001-5860-9490) ${ }^{2}$
${ }^{1}$ Curso de Fisioterapia, Universidade Federal de Goiás (UFGO). Rodovia BR-364, Setor Francisco Antônio. 75801-615 Jataí GO Brasil.

hmsfisio@yahoo.com.br

${ }^{2}$ Faculdade de Medicina,

UFGO. Goiânia GO Brasil.

${ }^{3}$ Faculdade de Matemática,

Universidade Federal de

Uberlândia. Uberlândia

MG Brasil.

\begin{abstract}
This study aimed to determine the impact of health on the quality of life (QoL) and quality of working life $(\mathrm{QWL})$ of university teachers. A cross-sectional study was conducted with 284 teachersin the areas of health, sciences, human sciences, and agricultural sciences using a sociodemographic questionnaire containing health-related questions, the WHOQOL-bref, and TQWL-42. Results: the findings showed that teachers who practiced physical activity had better QoL,QWL, and sleep quality. Advanced age was also associated with better QoL.Furthermore, QoL and QWL were lower in teachers who used medications, had an imbalanced diet, and did not practice leisure activities. QoL and QWL are influenced by health-related factors.
\end{abstract}

Key words Quality of life, Work, Illness, Teachers
Resumo O objetivo deste artigo é identificar o impacto da saúde na qualidade de vida (QV) e qualidade da vida profissional (QVT) de professores universitários. Estudo descritivo, transversal, que avaliou 284 professores universitários das áreas da saúde, biologia, agrarias, humanas e exatas. A avaliação ocorreu através da aplicação de um questionário sociodemográfico com questões relacionadas aos dados de saúde, o WHOQOL-bref para avaliar QV e o TQWL-42 para avaliar a qualidade da vida no trabalho (QVT). Verificouse que os professores que praticam atividade fisica apresentaram melhor $Q V$ e QVT e relataram melhor qualidade de sono, além disso, elas eram menores em professores que usam medicação, que têm uma dieta pobre e têm menos atividades de lazer. Além disso, a idade foi associada a melhor QV. A qualidade de vida e qualidade de vida no trabalho sofrem impacto dos aspectos relacionados à saúde.

Palavras-chave Qualidade de vida, Trabalho, Doença, Docentes 


\section{Introdução}

A docência é uma profissão cujo desgaste intelecto-emocional está sempre presente nos seus trabalhadores, expondo-os a riscos de saúde ${ }^{1,2}$ Trabalhar em ambientes e condições adversas, sem perspectivas profissionais, somado aos problemas pessoais, preocupa cada vez mais os docentes, visto que eles são potenciais candidatos ao desenvolvimento de doenças variadas, ligadas ou não ao estresse ${ }^{3}$.

Vários são os fatores que tornam a função de docente universitário propensa a doenças de cunho psicoemocional ou psicossomático, tais como as exigências referentes à alta produtividade científica, necessidade de constante atualização pela participação em congressos/ bancas, as longas jornadas de trabalho, inclusive nos finais de semana e feriados, falta de equipamentos e condições de trabalho, além do estresse tecnológico (caracterizado como uma reação do organismo com componentes psicológicos e físicos, que ocorre quando surge a necessidade inevitável de adaptação às importantes inovações tecnológicas), entre outros. Assim, a exigência e sobrecarga ao professor estende-se além da necessidade de atualização científica, promovendo nele culpa e sentimento de impotência por não conseguir suprir a demanda de tanta informação ${ }^{2,4}$.

A precarização do trabalho docente tem início nos anos 90, na conjuntura da globalização e das reformas neoliberais com políticas deliberadas que diluem as fronteiras entre o público e o privado, os brasileiros experienciam no campo do trabalho o acirramento das privatizações, a superexploração e a fragilização do poder sindical ${ }^{5}$. A organização do trabalho docente desde a década de 2000 aumenta ainda mais a precarização deste grupo de trabalhadores, exibindo formas flexibilizadas de contratação, além dos contratos temporários e efetivos baseados em horas-aula (pagamento por aula e ausência de Plano de Carreira), tem sido prática corrente o recurso às bolsas de pesquisa e adicionais por atividade de extensão como forma de remuneração docente apenas por aula ministrada, com menos tempo dedicado à pesquisa e extensão ${ }^{6}$.

Soma-se a isto o estímulo institucionalizado à concorrência interna, os professores voltam-se uns contra os outros, aprofundando o individualismo, neutralizando a mobilização coletiva. Um exemplo cada vez mais evidente no contexto universitário atual é a disputa individual, e não da unidade acadêmica, por cada mínimo recurso disponibilizado para pesquisas oferecido pelas agên- cias de fomento. Além da concorrência interna, têm sido implementados pelas políticas governamentais para orientar a população e ajudá-la a escolher "a melhor" instituição de ensino, avaliações técnicas, que se sustentam na necessidade de comparações e rankings e buscam o controle da qualidade dos serviços educacionais. Tais fatos aumentam a pressão sobre os professores e promovem aumento no uso de medicação e o adoecimento ${ }^{5}$.

Diante de tantas atribuições e demandas, algumas instituições de ensino superior ainda oferecem cursos/treinamento destinados à capacitação dos docentes universitários, uma vez que desse profissional se requer, em um nível mais complexo, uma atuação reflexiva, crítica e competente, sendo-lhe exigidas aulas interativas e que estimulem a aprendizagem dos alunos, compelindo-os a uma preparação/capacitação didática. Todavia, tal fato necessita do ensejo e disposição individual de cada docente e, aqueles que não se dispõem a tais adaptações e capacitação podem apresentar maiores dificuldades na execução da sua função, o que contribui para aumentar a exigência do docente dentro e fora do ambiente de trabalho e, por sua vez, pode afetar seu bem-estar em ambos ambientes?

O processo saúde-doença do docente também é construído no trabalho, de tríplice natureza - biológica, psicológica e social - interdependente e contraditória, fazendo com que o trabalho remeta a possibilidades variadas de consumo, satisfação, adoecimento e morte. Assim, o trabalho pode ser tido como um espaço de reafirmação da autoestima, de desenvolvimento de habilidades, de expressão das emoções, o que o torna um espaço de construção da história individual e de identidade social. Entretanto, o ambiente de trabalho pode produzir enfermidades ocupacionais, comprometendo a saúde física e mental do professor ${ }^{8}$.

Delcor et al. ${ }^{9}$ referem em seu estudo que $32,5 \%$ dos professores referiram problemas de saúde, sendo as queixas mais frequentes dores nas costas, dores nas pernas e problemas vocais, enquanto os distúrbios psicoemocionais mais comuns foram o nervosismo/ansiedade e cansaço mental. Apesar da grande exposição do docente universitário a problemas de saúde Lima e LimaFilho ${ }^{8}$ referem que pouca atenção é dada pelas autoridades governamentais e pelos dirigentes institucionais, o que culmina com um quadro crescente de mal-estar entre os docentes, tanto em termos físicos, psíquicos como interpessoais

A saúde é dimensionada também pela qualidade de vida $(\mathrm{QV})$, a qual abrange diversos 
aspectos, os quais abrangem a visão da intrínseca relação entre condições de trabalho e saúde, aproximando-se das visões clássicas da medicina social e da discussão que nos últimos anos vêm se revigorando na área e têm no conceito de promoção da saúde sua estratégia central. Tal conceito foi definido, tomando como base a concepção de que se consideram como os determinantes da saúde: o estilo de vida, os avanços da biologia humana, o ambiente físico e social e os serviços de saúde ${ }^{10,11}$.

Como a QV possui interferência ou interfere no âmbito da saúde, quando visto em sentido ampliado, ela se apoia na compreensão das necessidades humanas fundamentais, materiais e espirituais e, têm no conceito de promoção da saúde seu foco mais relevante, ressaltando que viver sem doença ou superar as dificuldades impostas por uma morbidade influenciam diretamente no entendimento da QV do indivíduo ${ }^{11}$.

Diante disso, o objetivo do presente estudo foi identificar a interferência de aspectos ligados a saúde na QV e QVT de docentes universitários das áreas da saúde, exatas, humanas e agrárias.

\section{Método}

Trata-se de um estudo observacional, descritivo, de corte transversal realizado nas dependências de uma instituição de ensino superior (IES) pública localizada em um estado do centro-oeste brasileiro. O presente estudo foi aprovado pelo Comitê de Ética em Pesquisa (CEP), respeitando-se os preceitos éticos de pesquisa envolvendo seres humanos. Todos os voluntários assinaram o Termo de Consentimento Livre e Esclarecido (TCLE).

A população docente na instituição de ensino superior analisada (IES) era de 386 servidores e a partir deste número foi realizado o cálculo amostral por intermédio do OpenEpi ${ }^{\circledR}$, utilizando nível de confiança de 95\%, resultou em uma amostra mínima de 193 docentes para uma representatividade em relação a toda a população da IES, todavia também foi feito o cálculo amostral por área do conhecimento/sexo e atingiu-se a representatividade em cada uma das áreas. Avaliou-se todas as áreas do conhecimento existentes na IES, afim de se ter uma visão geral das questões referentes à saúde, QV e QVT desta população. Foram incluídos no estudo os docentes com mais de seis meses de docência universitária independentemente de seu vínculo empregatício. Foram excluídos do estudo: questionários incompletos, portadores de deficiência física, docentes exercendo exclusivamente atividade administrativa ou em período de licença e docentes alunos de programas de pós-graduação Stricto Sensu em estágio de docência (Tabela 1).

A coleta de dados ocorreu simultaneamente em todos os cursos da IES. Para tanto, utilizou-se três instrumentos, um questionário sobre aspectos sociodemográficos com dados relacionados a saúde, um instrumento específico para avaliação da QVT, o Total Quality of Work Life (TQWL-42) e o instrumento genérico proposto pela Organização Mundial de Sáude (OMS) para avaliação da QV, denominado WHOQOL-Bref.

$\mathrm{O}$ primeiro instrumento corresponde a um questionário construído pelos próprios autores para coleta de dados sociodemográficos e relacionados à saúde. $\mathrm{O}$ questionário foi construído no formato estruturado e com os dados nominais categorizados. Após a sua elaboração ele foi refinado por 3 juízes, sendo estes, pesquisadores da área temática em questão ou experts no método proposto. Após o refinamento procederam-se as devidas correções e realizou-se dois pré-testes. Dos pré-testes culminou-se em adequações para que se obtivesse a versão final utilizada neste estudo.

O segundo instrumento, o TQWL-42, foi construído e validado por Pedroso et al. ${ }^{12}$ para quantificação da QVT em diferentes populações. Tal instrumento é fundamentado nos principais modelos clássicos de QVT encontrados na literatura e alicerçado no instrumento WHOQOL-100. Este instrumento possui 42 questões do tipo Likert e é dividido em 5 esferas: - Esfera 1: Biológica/Fisiológica; - Esfera 2: Psicológica/ Comportamental; - Esfera 3: Sociológica/Relacional; - Esfera 4: Econômica/Política; -Esfera 5: Ambiental/Organizacional.

Por fim, o terceiro instrumento, o WHOQOL -Bref, criado pela OMS em 1998, e validado para

Tabela 1. População e amostra $(\mathrm{N}=284)$.

\begin{tabular}{lcc}
\multicolumn{1}{c}{$\begin{array}{c}\text { Área } \\
\text { conhecimento }\end{array}$} & $\begin{array}{c}\text { Total de } \\
\text { professores }\end{array}$ & $\begin{array}{c}\text { Questionários } \\
\text { respondidos }\end{array}$ \\
\hline $\begin{array}{l}\text { Ciências biológicas } \\
\text { e da saúde }\end{array}$ & 158 & 113 \\
$\begin{array}{l}\text { Ciências agrárias e } \\
\text { da terra }\end{array}$ & 55 & 38 \\
Ciências exatas & 61 & 51 \\
Ciências humanas & 112 & 82 \\
Total & 386 & 284 \\
\hline
\end{tabular}


a língua portuguesa por Fleck et al. ${ }^{13}$ possui 26 questões do tipo Likert divididas em 4 domínios: - Domínio 1: físico; - Domínio 2: psicológico; - Domínio 3: relações sociais; Domínio: 4: ambiental.

Neste estudo, todos os questionários foram auto administrados, todavia em caso de dúvida, os pesquisadores estiveram à disposição para auxiliar os respondentes, convertendo a aplicação em assistida.

Para verificação da confiabilidade dos dois instrumentos na população estudada foi realizado o cálculo do Alfa de Cronbach, o qual determinou $\alpha=0,80$ para o TQWL-42 e $\alpha=0,85$ referente ao WHOQOL-Bref.

Para fins estatísticos, foram considerados apenas os questionários respondidos integralmente, no caso do WHOQOL-Bref e TQWL-42, enquanto para o questionário sociodemográfico foram aproveitados aqueles cujos dados utilizados no estudo estavam com as respostas completas, sendo descartados os demais. Nos casos em que um instrumento foi descartado os demais também o foram para aquele respondente.

Os dados coletados foram transferidos para uma planilha do Excel (Microsoft Office Excel ${ }^{\circledast}$ 2010) formando um banco de dados para realização da estatística descritiva e para um programa de análise estatística, sendo este, o software SPSS ${ }^{\circledast}$ (versão 22.0). Após a análise descritiva dos dados foi feito o teste de Shapiro-Wilks com a finalidade de determinar se os dados tinham distribuição normal. Feito isto, realizou-se a comparação da QV e QVT pelo o teste U de Mann-Whitney e o teste de Kruskal-Wallis, quando a comparação apresentava três ou mais grupos. Também foi realizado o Coeficiente de Correlação de Spearman para correlacionar os aspectos sociodemográficos quantitativos com os escores obtidos no TQWL-42 e no WHOQOL-Bref. Os resultados foram considerados estatisticamente significativos ao nível de $5 \%(\mathrm{p}<0,05)$.

\section{Resultados}

A amostra do presente estudo foi composta por 284 docentes, sendo 113 da área da saúde, 82 das humanas, 51 das exatas e 38 das agrárias. Chegou-se a tal amostra após excluírem-se 11 questionários por inadequação de preenchimento e 29 por enquadramento nos critérios de exclusão.

A Figura 1 apresenta o resultado da correlação entre idade e QV, a qual demonstrou correlação fraca e significante $(r=0,119$ e $p=0,045)$ entre as varáveis. A análise foi feita por meio do coeficiente de correlação de Spearman.

Na Tabela 2 estão apresentados os dados sociodemográficos dos docentes por área de conhecimento. Nas áreas exatas e agrárias há predomínio de docentes do sexo masculino e em todas as áreas houve dominância de docentes casados. Em relação a faixa etária, na área de saúde a maior parte dos docentes possuem entre 31-40 anos, enquanto na área das exatas é de 20-30 anos. Em oposição, as ciências agrárias possuem maior parte dos docentes com idade acima de 51 anos.

Os dados referentes às variáveis relacionadas a saúde são apresentados na Tabela 3. Observase que a maior parte dos docentes não possui doença diagnosticada, apesar disso os docentes das agrárias possuem maior número de docentes adoecidos. Em relação ao tabagismo e uso de bebida alcoólica, prevaleceram os não tabagistas e uso ocasional moderado de bebidas. A maior parte dos docentes realizam atividades de lazer de 1-2 vezes semanais, já em relação a prática de atividade física, o sedentarismo e a prática ocasional de baixa intensidade foram os mais relatados. Com relação à avaliação do sono, esta foi tida como boa ou regular por maior parte dos docentes avaliados (Tabela 3).

Na comparação da QV e QVT entre as categorias das variáveis relacionadas a saúde pode-se verificar algumas diferenças significantes. Foi verificado que a QV dos professores que já se afastaram do trabalho por motivo de doença $(\mathrm{QV}=14,56 \pm 1,71)$ apresentaram um escore geral significativamente menor $(\mathrm{p}=0,023)$ daqueles que nunca se afastaram $(\mathrm{QV}=15,29 \pm$ $1,74)$, o mesmo ocorreu em relação ao uso de medicamentos, de modo que os professores que fazem uso de medicamentos $(\mathrm{QV}=14,87 \pm 1,76)$ referiram um escore de QV estatisticamente menor $(\mathrm{p}=0,034)$, quando comparados àqueles que não o fazem $(\mathrm{QV}=15,35 \pm 1,72)$. Em relação a influência do lazer, constatou-se que os professores que referiram ter $1-2(\mathrm{QV}=15,55 \pm 1,77)$ ou 2-3 $(\mathrm{QV}=16,02 \pm 1,65)$ atividades de lazer por semana apresentaram uma melhor QV ( $\mathrm{p}=$ $0,001)$ do que aqueles que não tinham este tipo de atividade $(\mathrm{QV}=15,09 \pm 1,77)$ (Tabela 3$)$.

A prática de atividade física impactou tanto a QV quanto a QVT dos professores. Os docentes sedentários apresentaram uma QV $(\mathrm{QV}=14,65$ $\pm 1,75)$ e QVT $(\mathrm{QVT}=3,54 \pm 0,39)$ significativamente pior $(\mathrm{p}=0,006$ para $\mathrm{QV}$ e $\mathrm{p}=0,003$ para QVT) comparados aos docentes que praticavam atividade física regular de moderada intensidade $(\mathrm{QV}=15,38 \pm 1,62$ e $\mathrm{QVT}=3,27 \pm 0,42)$. Do 
mesmo modo que a atividade física, a qualidade do sono também teve reflexo na QV e QVT, sendo que em ambas os professores que referem ter um sono muito bom (QV $=15,85 \pm 1,74$ e QVT $=3,53 \pm 0,48)$ apresentaram uma percepção da QV e QVT significativamente melhor ( $\mathrm{p}=0,000$ para QV e $\mathrm{p}=0,000$ para QVT) comparados aos que referiram ter qualidade de sono regular $(\mathrm{QV}$ $=14,64 \pm 1,5$ e $\mathrm{QVT}=3,33 \pm 0,37)$ ou ruim $(\mathrm{QV}$ $=13,67 \pm 1,81$ e QVT $=3,22 \pm 0,43)$, assim como os que disseram ter qualidade de sono bom (QV $=15,75 \pm 1,50)$ apresentaram uma QV significativamente melhor $(\mathrm{p}=0,000)$ comparados àqueles com sono regular e sono ruim (Tabela 3 ).

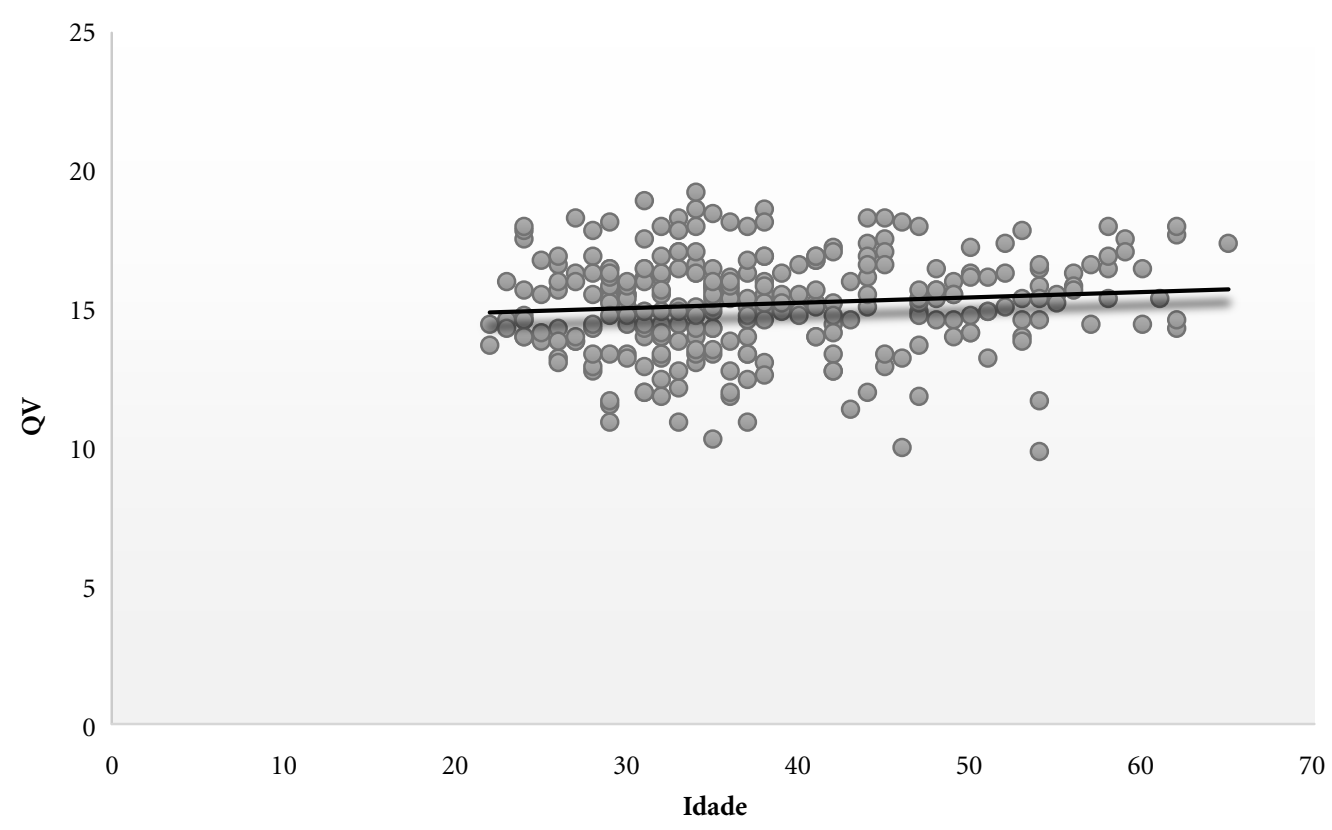

Figura 1. Correlação entre Idade e QV.

Tabela 2. Dados sociodemográficos distribuídos por área conhecimento $(\mathrm{N}=284)$.

\begin{tabular}{lcccc}
\hline \multicolumn{1}{c}{ Variável } & $\begin{array}{c}\text { Saúde } \\
(\mathbf{N}=\mathbf{1 1 3})\end{array}$ & $\begin{array}{c}\text { Humanas } \\
\mathbf{( N = 8 2 )}\end{array}$ & $\begin{array}{c}\text { Exatas } \\
\mathbf{N}=\mathbf{5 1})\end{array}$ & $\begin{array}{c}\text { Agrárias } \\
(\mathbf{N}=\mathbf{3 8})\end{array}$ \\
\hline $\begin{array}{l}\text { Sexo } \\
\text { Masculino }\end{array}$ & $50(44,2 \%)$ & $48(58,5 \%)$ & $40(78,4 \%)$ & $27(71,1 \%)$ \\
$\quad \begin{array}{l}\text { Feminino } \\
\text { Estado civil }\end{array}$ & $63(55,8 \%)$ & $34(41,5 \%)$ & $11(21,6 \%)$ & $11(28,9 \%)$ \\
$\quad$ Casado/União & $80(70,8 \%)$ & $52(46 \%)$ & $34(66,7 \%)$ & $27(71,1 \%)$ \\
Solteiro & $26(23 \%)$ & $21(25,6 \%)$ & $13(25,5 \%)$ & $8(21,1 \%)$ \\
Divorciado & $7(6,2 \%)$ & $1(1,2 \%)$ & $4(7,8 \%)$ & $2(5,3 \%)$ \\
Viúvo & $0(0 \%)$ & $8(9,8 \%)$ & $0(0 \%)$ & $1(2,6 \%)$ \\
Idade & & & & \\
$20-30$ & $24(21,2 \%)$ & $20(24,4 \%)$ & $21(41,2 \%)$ & $2(5,3 \%)$ \\
$31-40$ & $65(57,5 \%)$ & $24(29,3 \%)$ & $14(27,5 \%)$ & $11(28,9 \%)$ \\
$41-50$ & $14(12,4 \%)$ & $26(31,7 \%)$ & $11(21,6 \%)$ & $11(28,9 \%)$ \\
Acima de 51 & $10(8,8 \%)$ & $12(14,6 \%)$ & $5(9,8 \%)$ & $14(36,8 \%)$ \\
\hline
\end{tabular}




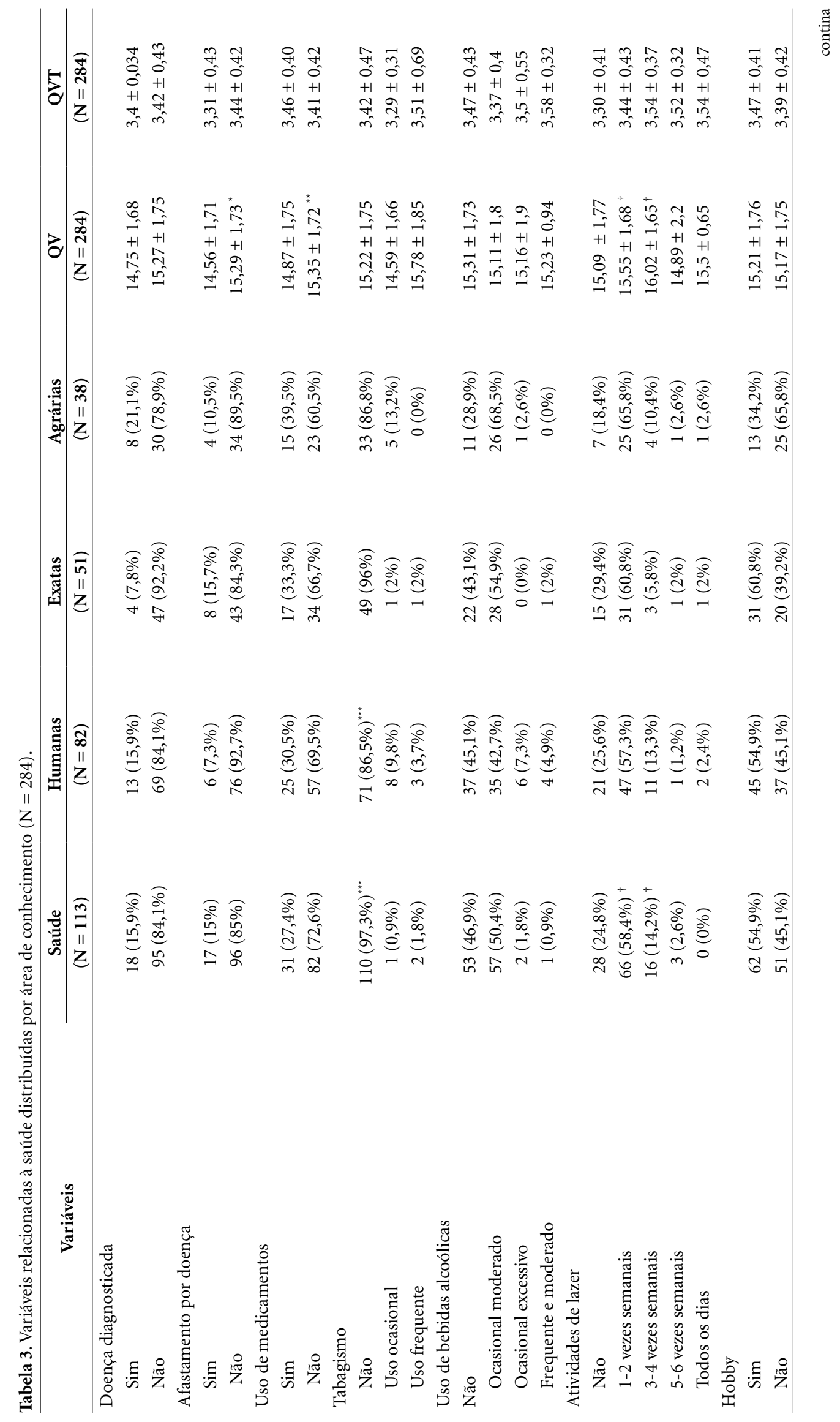




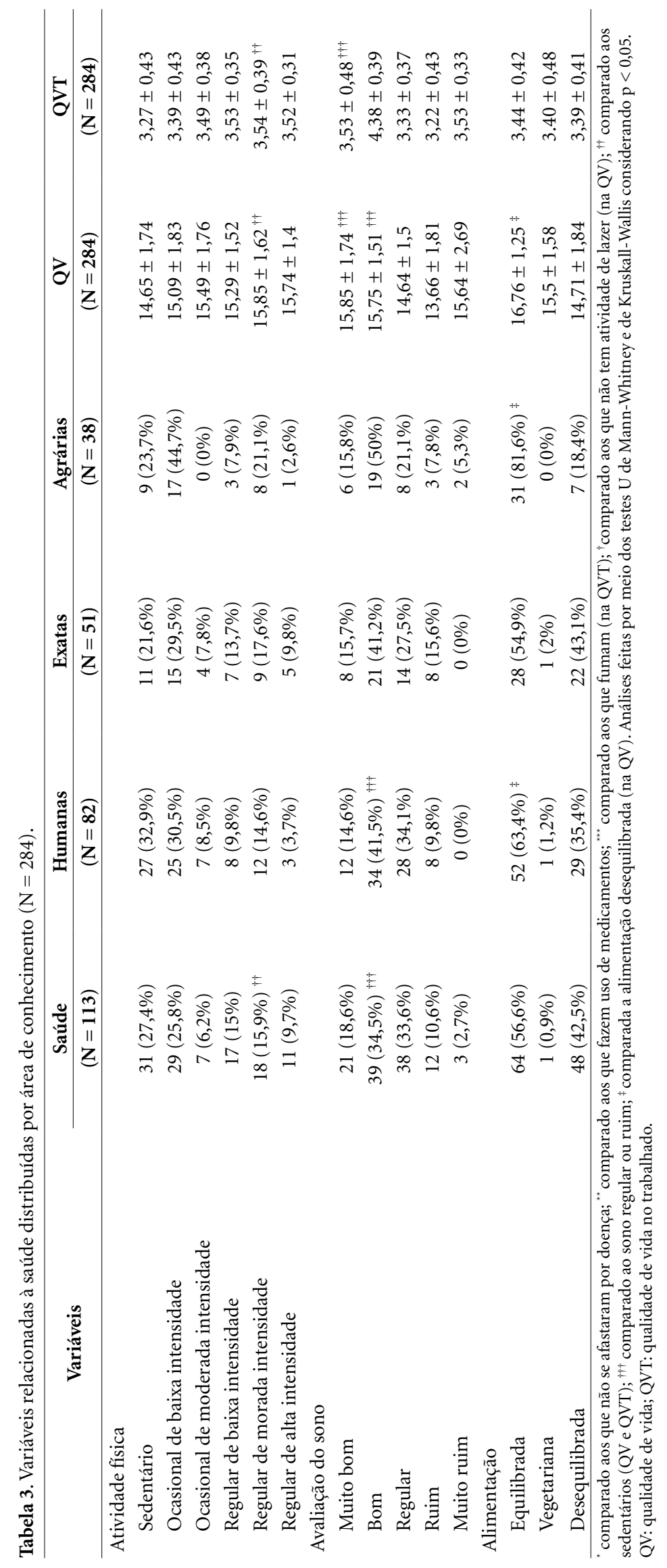


A alimentação também apresentou diferença na $\mathrm{QV}$ dos professores, visto que quando estes possuíam alimentação equilibrada $(\mathrm{QV}=15,5$ $\pm 1,58)$ sua $\mathrm{QV}$ foi significantemente maior $(\mathrm{p}=$ $0,000)$ do que aqueles com alimentação desequilibrada $(\mathrm{QV}=14,71 \pm 1,48)$, independentemente do tipo de desequilíbrio alimentar apontado (Tabela 3 ).

Na comparação entre as variáveis, realizada em cada área do conhecimento, verificou-se que os não tabagistas nas humanas (QVT $=3,45 \pm$ $0,39)$ e na saúde (QVT $=3,42 \pm 0,47)$ apresentaram uma QVT significantemente melhor $(\mathrm{p}=$ 0,012 tanto na saúde quanto nas humanas) comparados aos fumantes ocasionais $(\mathrm{QVT}=3,04 \pm$ 0,16 na saúde e $\mathrm{QVT}=3,01 \pm 1,38$ nas humanas). Os docentes que referiram ter um sono muito bom $(\mathrm{QV}=15,97 \pm 1,57$ e $\mathrm{QVT}=3,55 \pm 0,47)$ ou bom $(\mathrm{QV}=15,85 \pm 1,61 \mathrm{e} \mathrm{QVT}=3,55 \pm 0,45)$ na área da saúde e bom na humanas $(\mathrm{QV}=15,83$ $\pm 1,41)$ também referiram ter uma QV e/ou QVT significativamente melhor ( $\mathrm{p}=0,000$ na $\mathrm{QV}$ e QVT da saúde e $\mathrm{p}=0,002$ na QV da humanas) comparados àqueles que referiram ter um sono regular $(\mathrm{QV}=14,58 \pm 1,44, \mathrm{QVT}=3,25 \pm 0,20 \mathrm{na}$ saúde e $\mathrm{QV}=14,50 \pm 1,33$ na humanas) ou ruim $(\mathrm{QV}=12,92 \pm 1,80, \mathrm{QVT}=3,20 \pm 0,53$ na saúde $\mathrm{e} \mathrm{QV}=14,21 \pm 1,71$ na humanas). Em relação às atividades de lazer, constatou-se que os docentes da saúde que realizam 1-2 atividades de lazer semanal $(\mathrm{QV}=15,25 \pm 1,84$ e $\mathrm{QVT}=3,45 \pm 0,49)$ ou 2-3 (QV $=16,12 \pm 1,71$ e QVT $=3,56 \pm 0,36)$ atividades de lazer por semana, apresentaram uma melhor QV ( $\mathrm{p}=0,005)$ e QVT $(\mathrm{p}=0,008)$ do que aqueles que não tinham este tipo de atividade $(\mathrm{QV}=14,24 \pm 1,68 \mathrm{e} \mathrm{QVT}=3,18 \pm, 040)$.

$\mathrm{Na}$ comparação entre a atividade física verificou-se na área da saúde que os docentes cuja prática era regular de moderada intensidade $(\mathrm{QV}=$ $16,41 \pm 1,35$ e QVT $=3,61 \pm 0,40)$ sua QV e QVT foi estatisticamente maior, $\mathrm{p}=0,008$ e $\mathrm{p}=004$, respectivamente, comparados aos sedentários $(\mathrm{QV}=14,66 \pm 1,97$ e QVT $=3,24 \pm 0,49)$, aos que faziam atividades ocasionais de baixa intensidade $(\mathrm{QV}=14,73 \pm 1,98$ e $\mathrm{QVT}=3,36 \pm 0,51)$, ocasional de moderada intensidade $(\mathrm{QV}=15,08$ $\pm 1,77$ e $\mathrm{QVT}=3,32 \pm 0,44)$ e regular de baixa intensidade $(\mathrm{QV}=14,97 \pm 1,67)$. Por fim, verificou-se que os docentes com alimentação equilibrada nas agrárias $(\mathrm{QV}=15,84 \pm 1,07)$ e humanas $(\mathrm{QV}=15,67 \pm 1,53)$ possuíram uma $\mathrm{QV}$ estatisticamente maior $(\mathrm{p}=0,030$ nas agrárias e $\mathrm{p}$ $=0,007$ nas humanas) comparado a alimentação desequilibrada $(\mathrm{QV}=14,68 \pm 1,36$, nas agrárias e $\mathrm{QV}=14,44 \pm 1,54$, nas humanas).

\section{Discussão}

A carreira de docência universitária se faz por grande empenho e comprometimento por parte dos docentes, todavia as peculiaridades inerentes a tal função interferem negativamente em aspectos ligados à saúde com reflexo na QV e QVT do docente, conforme verificado no atual estudo, tal como baixa qualidade do sono, poucas atividades de lazer, baixo índice de atividade física e má alimentação.

O trabalho é salutar para a vida e sobrevivência, além de ser fonte de realização, satisfação, QV e mesmo saúde. Entretanto, ele também pode se transformar em elemento patogênico, tornandose nocivo à saúde, a depender do tipo de trabalho, do ambiente, das condições e da maneira como está organizado ${ }^{2,9}$.

Como em outras atividades profissionais, o comprometimento da saúde dos professores pode ser associado com diversos aspectos e relaciona-se à sua baixa QV. Desta forma, é importante aprofundar o conhecimento sobre a saúde em professores, explorando mecanismos biológicos, ergonômicos, ocupacionais e psicossociais de ensino, bem como investir em práticas que melhoram a relação de convivência entre os trabalhadores $^{14,15}$.

A fadiga emocional e mental desenvolvida nesse cotidiano os tornam frágeis, irritados e angustiados, alterando as necessidades fisiológicas relacionadas ao descanso físico e mental. Os professores consideram que trabalhar com pessoas é muito desgastante para a saúde, seja pelo estresse provocado pelas interações diretas com os alunos e a população assistida, seja pelas demais interações no contexto institucional, especialmente aquelas que determinam atitudes que contrariam seus princípios e disponibilidades. Eles próprios têm consciência de que essas situações provocam baixa imunidade e repercussões nas suas condições de saúde geral, favorecendo estados gripais constantes, enxaquecas, labirintites, crises hipertensivas, estados depressivos, problemas dermatológicos, entre outros ${ }^{16}$.

Este fato também pode ser explicado pela precarização e organização contemporânea do trabalho docente, visto que nota-se nas universidades a predominância de uma nova realidade: a demissão de professores doutores de mais larga experiência, cedendo lugar à contratação de especialistas e graduados; a rotatividade de docentes contratados temporariamente; a ênfase na lógica "menos professores, mais alunos"; o rebaixamento das remunerações, sem perspectivas 
de recuperação de perdas salariais; o desempenho acadêmico baseado na lógica produtivista, a qual dificulta a realização de práticas que visam à transformação social e à indissociabilidade entre ensino, pesquisa e extensão. Ainda versando sobre o fato das baixas remunerações, o docente vincula-se a diferentes instituições de ensino, simultaneamente, ministrando várias disciplinas (algumas delas incompatíveis com a sua formação acadêmica) e deslocando-se de um extremo a outro para poder cumprir a sua jornada de trabalho. Todos esses elementos impactam o trabalho docente, com consequências inquestionáveis sobre a saúde $e^{6,17}$.

Tais afirmações corroboram com o achado do presente estudo, segundo o qual professores universitários com histórico de afastamento por motivos diversos de saúde apresentam uma pior QV. Além dos fatores citados acima, o docente quando deixa sua função por problemas de saúde, ao regressar às atividades acadêmicas tem que retomar toda a rotina de atividades extra aula, e concomitante a isto, diversos fatores impactam sobre esta fase e sobre a QV, tais como, atualização de conhecimentos, envolvimento com novos colegas de trabalho e chefia, novas funções, mudança de rotina, mudança nos horários de trabalho, readaptação ao convívio com alunos, dentre outros ${ }^{6}$.

Muitos docentes não se afastam das atividades, porém fazem uso de medicamentos. Tal fato foi associado a uma pior QV. Todavia verificou-se que grande parte dos que utilizam de medicação, não referiu nenhuma doença diagnosticada, ou seja, se automedicam. A automedicação é definida como a seleção e uso de drogas por pessoas, ou membros da família do indivíduo medicado, para tratamento de condições ou sintomas autodiagnosticados ${ }^{18}$. Esta atitude pode incorrer em alguns prejuízos ou riscos, tais como autodiagnostico incorreto, demora na busca por atendimento de um profissional de saúde, reações adversas, interações entre diferentes drogas, modo incorreto de uso/dosagem, escolha incorreta de drogas, mascaramento de doenças mais graves e risco de dependência e/ou abuso ${ }^{18,19}$.

O presente estudo não se propôs analisar os motivos e como ocorre esta automedicação. Todavia, pela pluralidade de fatores inerentes à docência universitária, pode-se inferir que este fato se associa principalmente a três fatores: falta de tempo para o cuidado ideal com a saúde, ansiedade e quadros álgicos constantes ${ }^{8,16,20-22}$.

As dores musculoesqueléticas são comuns na população docente, acometendo entre 90-100\% desta classe de trabalhadores, esta alta prevalência pode ser explicada pela posição adotada por estes profissionais ao exercerem sua atividade, com o ombro em elevação e postura ortostática ou sentada por longos períodos ${ }^{15,21-24}$.

Outro fator que pode desencadear ou piorar o quadro de mialgia é a ansiedade, em decorrência do aumento da tensão muscular. Outro fator que pode estar ligado à ansiedade está relacionado a precarização e organização atual do trabalho docente, os quais os expõe a conflitos e tensões da cotidianidade do trabalho que emergem complexos movimentos de fuga, mas também de enfrentamento e ruptura, os quais promovem o dissenso, ligados ao compromisso social coletivo e o devido reconhecimento dos direitos fundamentais de todo e qualquer ser humano à cidadania e de receber educação de qualidade, enquanto padecem com a falta de reconhecimento governamental, social, baixas remunerações, ausência de plano de carreira, falta de estabilidade empregatícia e necessidade de ter mais de um emprego para garantir melhor renda e saúde $e^{6,7,17}$.

A ansiedade é um sintoma que comumente acompanha quadros depressivos e de estresse excessivos, ela é definida como uma sensação vaga e difusa, desagradável, de apreensão ou tensão, que se acompanha de diversas manifestações físicas, tais como, dispneia, taquicardia, tensão muscular, sudorese, tremor, dentro outros ${ }^{25-28}$.

Verificou-se ainda que os docentes das áreas da saúde e humanas que fazem uso de tabaco apresentaram um menor escore de QV, isto também pode ser uma justificativa acerca da ansiedade e do estresse, visto que os profissionais desta área lidam com situações de angústia e responsabilidades inerentes às suas atividades. No entanto, verificou-se no atual estudo que os tabagistas apresentaram uma pior QV. Embora muitos fumantes não tenham problemas de saúde evidentes, os resultados da combustão do tabaco na produção de agentes tóxicos são potencialmente prejudiciais. Assim, fumantes têm pior saúde física, maior referência de algias, menos vitalidade, alterações emocionais e mentais (depressão, baixa autoestima, e uma predisposição para a adoção de um estilo de vida não saudável) em comparação com não fumantes ${ }^{29,30}$.

O tabagismo tem um efeito sobre a percepção de saúde, independentemente dos seus efeitos somáticos. Por exemplo, o tabagismo está associado a situações de estresse e dificuldade de adaptação social, o que por sua vez pode afetar a percepção da QV. A falta de vitalidade dos tabagistas pode ser explicada pela disfunção pulmonar que 
ocorre pela hipersecreção mucosa, capacidade de difusão reduzida, aumento do monóxido de carbono nos pulmões e obstrução do fluxo de ar periférico. Outro mecanismo possível para explicar as diferenças na QV dos fumantes são as lesões musculoesqueléticas, devido à vasoconstrição e hipóxia que prejudicam a perfusão e nutrição destes tecidos ${ }^{29-31}$.

Contribui também para uma pior QV e QVT a má qualidade do sono, conforme revelado pelo atual estudo. Os professores com pior qualidade do sono apresentam menores índices de QV e QVT. Segundo Souza e Reimão ${ }^{32}$ a má qualidade do sono, ou a insônia, afeta a QV de seus portadores; afirmam ainda que, quando a insônia é crônica, geralmente reflete distúrbios psicológicos e comportamentais.

No estudo de Souza et al. ${ }^{33}$ verificou-se que $46 \%$ a $51 \%$ dos docentes avaliados apresentaram sonolência diurna excessiva e má qualidade do sono, tais fatos podem comprometer a saúde e $\mathrm{QV}$, além de influenciar no desempenho da atividade docente, o que pode afetar a educação de seus alunos. Tal fato se dá por diversos motivos, dentre eles as demandas sociais, níveis de ruído e características de ocupação da profissão docente (excesso de trabalho, alto índice de estresse, atividades extras sala, fatores organizacionais, dentre outros) $)^{33,34}$. Além de o sono interferir na QV, especificamente para os docentes, ele também influencia negativamente para a qualidade vocal, prejudicando este profissional na execução da sua função e diminui sua capacidade produtiva ${ }^{35}$.

Além da ansiedade, depressão, dores musculoesqueléticas e estresse, outros fatores apresentados no atual estudo corroboram com a perda ou má qualidade do sono referida pelos docentes avaliados, visto que aqueles com menor frequência de atividades de lazer, de atividade física e com má alimentação também apresentaram uma menor QV.

Os docentes das áreas da saúde e humanas que referiram uma boa qualidade do sono também possuíram uma melhor QV. Tais docentes também foram os que menos referiram fazer uso de medicamentos e possuem atividades de lazer mais frequentes. Além disso, os docentes da saúde ainda referiram uma melhor QV quando praticavam atividade física de moderada intensidade e, a atividade física auxilia na diminuição do estresse, propiciando benefícios a todos os sistemas orgânicos, por meio da liberação de opioides endógenos que propiciam bem-estar e relaxamento ${ }^{36,37}$. Já os docentes das humanas, assim como os das agrárias, apresentaram uma QV melhor quando tinham sua alimentação equilibrada, a qual também mantém um equilíbrio orgânico e auxilia o $\operatorname{sono}^{38}$. Além dos fatores já mencionados, não se pode ignorar que as perdas coletivas referentes a remuneração, desvalorização, diminuição das verbas e auxilio para pesquisa e extensão, alterem o estado emocional dos docentes e impactam negativamente no sono ${ }^{7}$.

Outro fator que influencia a QV é o estilo de vida, sendo que o sedentarismo é a principal causa de saúde debilitada para um grande número de indivíduos. $O$ fato de adicionar atividade física regular ao estilo de vida desses indivíduos já proporciona melhoras substanciais na saúde global e na $\mathrm{QV}$, conforme relatado pelos docentes da saúde no atual estudo ${ }^{36,37}$.

Além disso, a prática de atividade física é tida por algumas pessoas como uma atividade de lazer e contribui de maneira positiva para a promoção da saúde e apresenta associação positiva com a $\mathrm{QV}^{36}$. Ademais, a melhora da $\mathrm{QV}$ também é percebida quando a prática da atividade física busca a prevenção/promoção, a reabilitação, ou gestão da doença, principalmente porque indivíduos que praticam atividade física possuem uma melhor resposta orgânica contra doenças e afecções, as quais podem diminuir a capacidade funcional e laboral das pessoas ${ }^{39,40}$. A atividade física também promove estímulos ao sistema muscular, o que por sua vez melhora o equilíbrio de forças atuantes nas articulações, diminuindo a sobrecarga imposta a elas, com consequente diminuição de algias e melhora do bem-estar do indivíduo ${ }^{41}$.

Além disso, a regularidade na prática de exercícios promove benefícios emocionais, promovidos pelo aumento na produção e liberação de endorfinas pelo sistema nervoso central, fato este que influencia na diminuição do estresse, da ansiedade, da depressão, da tensão emocional e, ainda, melhora a socialização, a qualidade do sono, o bem-estar e, portanto, a QV dos indivíduos $^{39}$.

A QV dos docentes poderia ser melhor caso não tivessem um grande número de atividades a eles impostas e uma carga horária semanal de trabalho que dificultasse atividades relacionadas a lazer e atividades recreativas. Estes problemas podem agravar a exaustão física e psicológica provocada aos professores ${ }^{42}$. A participação em atividades de lazer proporciona aos participantes mais disposição, maior agilidade mental, reduz o estresse e permite uma melhor gestão do tempo. Além disso, o professor possui uma relação positiva com a saúde, apresenta não apenas ausência 
de doenças, mas mostra mais capacidade de aumentar a satisfação com a vida e melhor gestão do estresse. Soma-se a isso o fato das relações sociais produzirem um efeito anti-estresse e de satisfação às pessoas ${ }^{43}$.

Diante do exposto, salienta-se a necessidade de um olhar mais criterioso e minucioso acerca dos fatores que afetam a QV e, por consequência, a saúde dos docentes universitários, visto que tal profissão exige muito das capacidades físicas, intelectuais, sentimentais e emocionais destes profissionais.

\section{Colaboradores}

HM Sanchez e EGM Sanchez: concepção e o delineamento, a análise e interpretação dos dados, redação do artigo ou a sua revisão crítica e aprovação da versão a ser publicada; EC Guimarães: delineamento, a análise e interpretação dos dados, revisão crítica; MA Barbosa e CC Porto: concepção, o delineamento, interpretação dos dados, sua revisão crítica.

\section{Conclusão}

Os docentes universitários das áreas da saúde, exatas, humanas e agrárias possuem aspectos ligados à saúde que interferem negativamente na sua QV e QVT, à exemplo da pior qualidade do sono, do sedentarismo, poucas atividades de lazer, do uso de medicamentos (automedicação), do afastamento por doenças e da alimentação desequilibrada. Além disso, a maior idade está associada a melhores escores de QV nos docentes universitários.

\section{Referências}

1. Bureau International du Travail (BIT). Emploi et conditions de travail dês ensaignants. Geneve: BIT; 1981.

2. Bogaert I, De Martelaer K, Deforche B, Clarys P, Zinzen E. Associations between different types of physical activity and teacher's perceived mental, physical, and work-related health. BMC Public Health 2014; 14(534):1-9.

3. Kovess-Masféty V, Sevilla-Dedieu C, Rios-Seidel C, Nerrière E, Chan Chee C. Do teachers have more health problems? Results from a French cross-sectional survey. BMC Public Health 2006; 6(101):1-13.

4. Silva AA, Fischer FM. Teacher's sick leave due to mental and behavioral disorders and return to work. Work 2012; 41:5815-5818.

5. Santos SDM. A precarização do trabalho docente no Ensino Superior: dos impasses às possibilidades de mudanças. Educar em Revista 2012; 46:229-244.

6. Bosi AP. A precarização do trabalho docente nas Instituições de Ensino Superior do Brasil nesses últimos 25 anos. Educ. Soc. 2007; 28(101):1503-1523.

7. Anastasiou GC, Pimenta SG. Docência no ensino superior. São Paulo: Cortez; 2002.

8. Lima MFEM, Lima-Filho DO. Work and health conditions of university professors. Ciências \& Cognição 2009; 14(3):62-82.

9. Delcor NS, Araújo TM, Reis EJFB, Porto LA, Carvalho FM, Silva MO, Barbalho L, Andrade JM. Condições de trabalho e saúde dos professores da rede particular de ensino de Vitória da Conquista, Bahia, Brasil. Cad Saud Publica 2004; 20(1):187-196.

10. Martin AJ, Stockler M. Quality of life assessment in health care research and practice. Evaluation \& Health Professions 1998; 21(2):141-156.

11. Minayo MCS, Hartz ZMA, Buss PM. Qualidade de vida e saúde: um debate necessário. Cien Saude Colet 2000; 5(1):7-18.

12. Pedroso B, Pilatti LA, Gutierrez GL, Picinin CT. Construction and validation of TQWL-42: a quality of work life measurement instrument. Revista de Salud Publica 2014; 16:885-896. 
13. Fleck MPA, Louzada S, Xavier M, Chachamovich E, Vieira G, Santos L, Pinzon V. Aplicação da versão em português do instrumento abreviado de avaliação da qualidade de vida "WHOQOL-Bref”. Rev Saude Publica 2000; 34(2):178-183.

14. Horn JE, Taris TW, Schaufeli WB, Schreurs PJG. The structure of occupational well-being: a study among Dutch teachers. J Occup Organ Psychol 2004; 77:365375.

15. Ceballos AGC, Santos GB. Factors associated with musculoskeletal pain among teachers: sociodemographics aspects, general health and well-being at work. Rev. bras. epidemiol. 2015; 18(3):702-715.

16. Silvério MR, Patrício ZM, Brodbeck IM, Grosseman S. O ensino na área da saúde e sua repercussão na qualidade de vida docente. Revista Brasileira de Educação Médica 2010; 34(1):65-73.

17. Gaspar RF, Fernandes TC. Oligopolização e precarização do trabalho docente no ensino superior privado brasileiro: causas, conexões e consequências. Rev Espaço Acadêmico 2015; 168:77-92.

18. Ruiz ME. Risks of self-medication practices. Curr Drug Saf 2010; 5(4):315-323.

19. Hughes CM, Mcelnay JC, Fleming GF. Benefits and risks of self medication. Drug Saf 2001; 24(14):10271037.

20. Kumar D, Deo JM. Stress and work life of college teachers. Journal of the Indian Academe of Applied Psychology 2011; 37:78-85.

21. Koetz L, Rempel C, Périco E. Quality of life of professors of higher education community institutions in the state of Rio Grande do Sul, Brazil. Cien Saude Colet 2013; 18(4):1019-1028.

22. Sanchez HM, Gusatti N, Sanchez EGM, Barbosa MA. Incidência de dor musculoesquelética em docentes universitários. Rev Bras Med Trab 2013; 11(2):66-75.

23. Carvalho AJFP, Alexandre NMC. Sintomas osteomusculares em professores do ensino fundamental. Rev Bras Fisioter 2006; 10(1):35-41.

24. Cardoso JP. Prevalência de dor musculoesquelética em professores. Rev Bras Epidemiol 2009; 12(4):604614.

25. Cheniaux E. Manual de Psicopatologia. 5a ed. Rio de Janeiro: Guanabara Koogan; 2015.

26. Zurlo MC, Pes D, Cooper CL. Stress in teaching: A study of occupational stress and it's determinants among Italian school teachers. Stress and Health 2007; 23:231-241.

27. Stoeber J, Rennert D. Perfectionism in school teachers: Relations with stress appraisals, coping styles, and burnout. Anxiety, Stress, \& Coping 2008; 21(1):37-53.

28. Robinson JA, Sareen J, Cox BJ, Bolton JM. Correlates of self-medication for anxiety disorders: results from the National Epidemiolgic Survey on Alcohol and Related Conditions. J Nerv Ment Dis 2009; 197(12):873878.

29. Cayuela A, Rodríguez-DomínguezS, Otero R. Deteriorated Health-Related Quality of Life in Healthy Male Smokers. Arch Bronconeumol 2007; 43:59-63.

30. Kiyohara K, Itani Y, Kawamura T, Matsumoto Y, TakahashiY. Changes in the SF-8 scores among healthy non-smoking school teachers after then forcement of smoke-free school policy: a comparison by passive smoke status. Health and Quality of Life Outcomes 2010; 8(44):2-8.
31. Tomioka H, Sekiya R, Nishio C, Ishimoto G. Impact of smoking cessation therapy on health-related quality of life. BMJ Open Resp Res 2014; 1:1-9.

32. Souza JC, Reimão R. Epidemiologia da insônia. Psicol Estud 2004; 9(1):3-7.

33. Souza JC, Sousa IC, Belísio AS, Azevedo CVM. Sleep habits, daytime sleepiness and sleep quality of high school teachers. Psychol Neurosci 2012; 5(2):257-263.

34. Qin J, Liu W, Zhu J, Weng W, Xu J, Ai Z. Health Related Quality of Life and Influencing Factors among Welders. PLoS One 2014; 9(7):e101982.

35. Pizolato RA, Rehder MI, Meneghim MC, Ambrosano GM, Mialhe FL, Pereira AC. Impact on quality of life in teachers after educational actions for prevention of voice disorders: a longitudinal study. Health Qual Life Outcomes 2013; 11:28.

36. Pucci GCMF, Rech CR, Fermino RC, Reis RS. Associação entre atividade física e qualidade de vida em adultos. Rev Saude Publica 2012; 46(1):166-179.

37. Gill DL, Hammond CC, Reifsteck EJ, Jehu CM, Williams RA, Adams MM, Lange EH, Becofsky K, Rodriguez E, Shang YT. Physical Activity and Quality of Life. J Prev Med Public Health 2013; 46(1):28-34.

38. Crispim CA, Zimberg IZ, Dos Reis BG, Diniz RM, Tufik S, Mello MT. Relationship between Food Intake and Sleep Pattern in Healthy Individuals. J Clin Sleep Med 2011; 7(6):659-664.

39. Penedo FJ, Dahn JR. Exercise and Well-Being: A Review of Mental and Physical Health Benefits Associated With Physical Activity. Curr Opin Psychiatry 2005; 18(2):189-193.

40. Gillison FB, Skevington SM, Sato A, Standage M, Evangelidou S. The effects of exercise interventions on quality of life in clinical and healthy populations; a meta-analysis. Soc Sci Med 2009; 68(9):1700-1710.

41. Abell JE, Hootman JM, Zack MM, Moriarty D, Helmick CG. Evidence based public health policy and practice: Physical activity and health related quality of life among people with arthritis. J Epidemiol Community Health 2005; 59(5):380-385.

42. Fernandes MH, Rocha VM. Impact of the psychosocial aspects of work on the quality of life of teachers. Rev Bras Psiquiatr 2009; 31(1):15-20.

43. Ajibua MA, Olorunsola HK, Bewaji OB. Perceived motivational factors influencing leisure-time physical activity involvement of teaching and nonteaching staff in tertiary institutions in Ondo state, Nigeria. Inter J Asian Soc Sci 2013; 3(1):10-19.

Artigo apresentado em 01/08/2017

Aprovado em 07/04/2018

Versão final apresentada em 09/04/2018 